\title{
Donne in Accademia
}

\section{Annalisa Nesi}

PUBBLICATO: 06 OCTOBER 2020

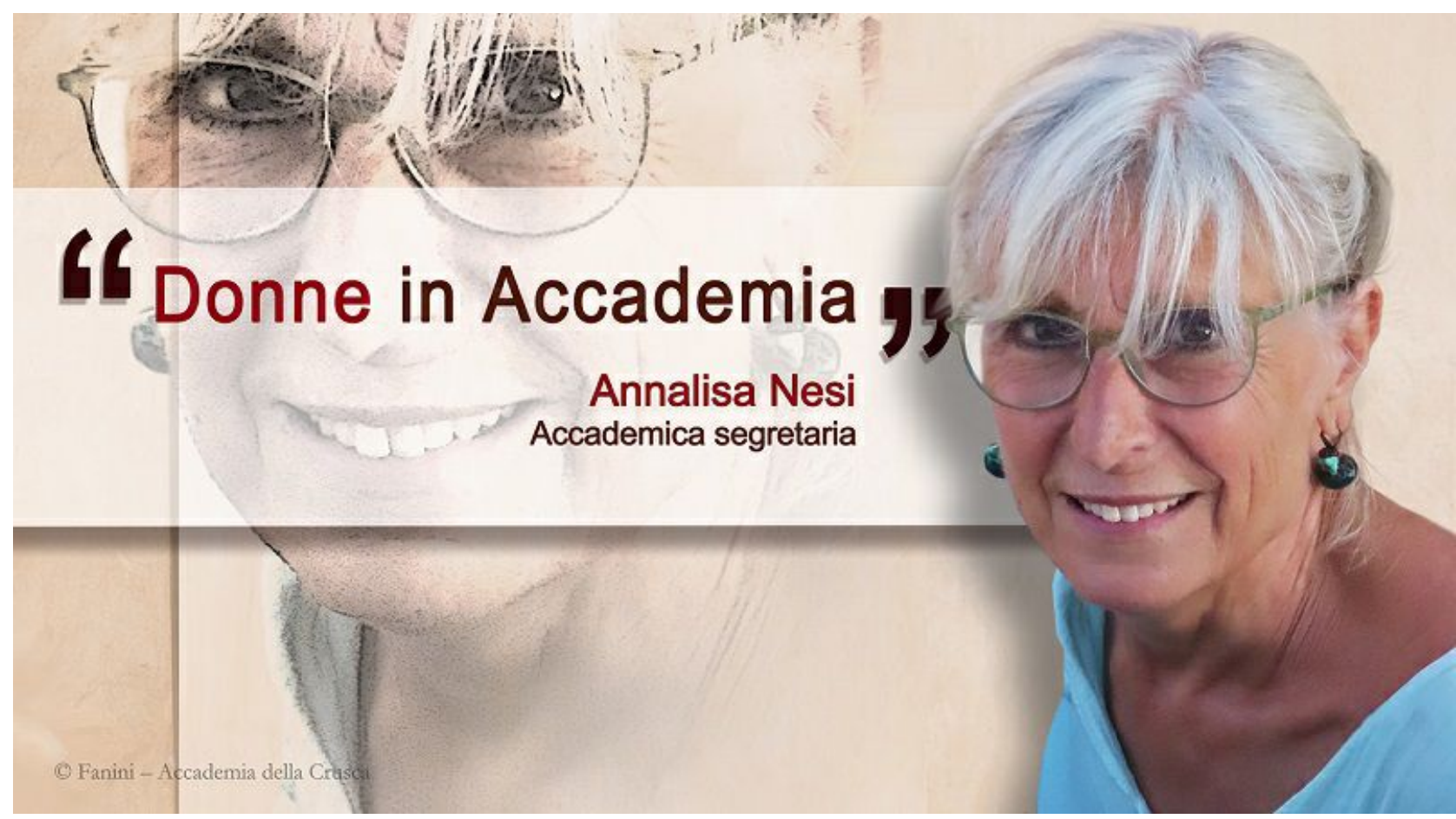

$\mathbf{L}^{\prime}$

Accademia è stata fondata e guidata per molti secoli da uomini che hanno ricoperto le varie cariche istituite a partire dal 1589 . Prima di entrare nel tema che tratteremo, cioè la presenza delle donne nell'Accademia oggi e nel passato, ritengo opportuno soffermarmi un attimo sulle regole di funzionamento della nostra istituzione, fissate da una normativa approvata dal ministero da cui dipendiamo, cioè il MIBACT, il Ministero per i Beni culturali.

Il presidente guida l'Accademia con l'aiuto del Consiglio direttivo, formato dal presidente stesso e da quattro accademici nei ruoli di vicepresidente, accademico segretario, consiglieri. Il Collegio, che, come si legge nello Statuto, ha il compito "di garantire la continuità della vita dell'Accademia e di determinare lo sviluppo delle sue attività scientifiche e culturali", è composto da tutti gli accademici ordinari (ad oggi 42) che eleggono il presidente e nominano i quattro consiglieri del Consiglio direttivo. Tutti gli accademici vengono eletti dal Collegio dopo essere stati candidati da almeno 5 membri. Vi sono inoltre gli accademici corrispondenti, divisi in italiani (I9) e esteri (29), che vengono aggregati con le stesse modalità degli accademici ordinari. L'Accademia è un ente pubblico con autonomia scientifica e organizzativa che sceglie i propri membri attraverso l'aggregazione; si avvale del Collegio dei revisori dei conti per il controllo legale e contabile della gestione finanziaria, composto da un membro nominato dal Collegio degli accademici, uno dal MIBACT e uno dal Ministero dell'Economia e delle Finanze.

Nel quadro dell'attuale organizzazione soltanto un concorso di forze che operano in sinergia permette il raggiungimento degli obiettivi che si sono via via aggiunti a quello storico dell'attività lessicografica: la formazione alla ricerca, l'educazione linguistica e il rapporto con la scuola, la consulenza linguistica, luogo privilegiato del dialogo con un vasto pubblico che pone domande sulla lingua e a cui è rivolto 
anche il periodico "La Crusca per voi". Agli accademici, la cui attività è a titolo gratuito, si devono aggiungere non solo il personale dell'amministrazione, della segreteria e della biblioteca - l'organico attualmente è di 7 persone - ma anche circa 20 collaboratori precari che operano a tutti i livelli, soprattutto borsisti, assegnisti, giovani in formazione e del Servizio civile. Dunque in molti animano l'attività odierna con impegno, capacità e soprattutto con passione e dedizione.

La presenza femminile è via via divenuta più consistente sia fra gli accademici sia fra il personale e i collaboratori. Comunque se le donne sono ancora poche nelle posizioni apicali - una sola nell'attuale Consiglio direttivo - sono invece molte nella veste di collaboratrici.

Si deve sottolineare che l'accesso delle donne alla Crusca ha un inizio precoce rispetto ad altre accademie europee e italiane: siamo nel 1978 quando viene eletta la prima donna alla Real Academia Española, la poetessa Carmen Conde Abellán (1907 - 1996), e di li a due anni, nel I980, entra a far parte dell'Académie française la scrittrice Marguerite Yourcenar (I903-1987). Circa un secolo prima, nel I879, l'Accademia dei Lincei aveva aperto le sue porte all'archeologa e epigrafista Ersilia Caetani Lovatelli (I840-I925) che diviene socia corrispondente dell'Accademia della Crusca qualche anno dopo, nel I893. È tuttavia la seconda donna in Accademia, infatti il primato va a Caterina Franceschi Ferrucci (I803-I887), letterata, poetessa e patriota, eletta col massimo dei voti socia corrispondente nel ı87r. Oltre quanto oggettivamente le viene riconosciuto dagli studi letterari e linguistici a quelli pedagogici, all'impegno per l'educazione delle giovani donne, Caterina Franceschi Ferrucci è figura emblematica del nuovo ruolo delle donne nell'Italia post-unitaria che, conquistati con fatica "i diritti politici e l'accesso ai nuovi spazi civili e professionali", sapranno "farsi valere nel mondo delle scienze, della letteratura, della ricerca pedagogica e della scuola, con evidenti e durature conseguenze sulle vicende linguistiche post-unitarie".

Nella storia dell'Accademia da quel I3 giugno I87I, a parte Caetani Lovatelli, si deve attendere il I970 per l'elezione della filologa Franca Brambilla Ageno che già dal 1939 aveva iniziato la sua collaborazione al Centro di studi di filologia italiana, ottenendo un ottimo giudizio per ricoprire l'incarico. Questo stesso Centro è stato molti anni dopo, dal 2000 al 20I2, diretto da unaltra illustre filologa, Rosanna Bettarini, socia corrispondente dal 1977, poi accademica dal 1988 e membro del Consiglio direttivo dal 2008 al 20r2. Ancora negli anni Settanta (1974) Tatjana Alisova, che insegnava lingue romanze all'Università di Mosca, entra all'Accademia come prima studiosa nel novero dei soci corrispondenti esteri. Dal 1972 al 2000 con la presidenza di Giovanni Nencioni il numero delle studiose elette accademiche e socie arriva a I2: Severina Parodi (1983; 1995), la già citata Rosanna Bettarini, le storiche della lingua (in ordine di prima nomina come socie) Maria Corti (1976; I989), Teresa Poggi Salani (ı988; I995), Maria Luisa Altieri Biagi (ı988; 1997), Ornella Castellani Pollidori (1990; 1995), Nicoletta Maraschio (1995; 1997), Silvia Morgana (1997; 2010), la linguista Bice Mortara Garavelli (I99I; 1999), la dialettologa Gabriella Giacomelli (1999), la storica dell'arte Paola Barocchi (I990; I995) e nel novero delle socie estere Jacqueline Brunet, studiosa di lingua e letteratura italiana. È a partire da questa rosa di accademiche che le donne di Crusca cominciano a ricoprire incarichi e cariche in cui comparivano solo accademici fino all'elezione, dopo oltre un secolo dall'ammissione della prima socia, di Nicoletta Maraschio, prima presidente, dal 2008 al 2014 e ora presidente onoraria, già membro del Consiglio direttivo (I997-2014) e vicepresidente durante la Presidenza di Francesco Sabatini (2000 al 2008). Durante il suo mandato aumenta il numero delle accademiche e continuerà a crescere anche dopo. In altre istituzioni come l'Accademia dei Lincei, che comunque ha accresciuto il numero delle accademiche, la Dante Alighieri o l'Istituto dell'Enciclopedia italiana nessuna donna ha ricoperto la carica di presidente o ruoli apicali.

La presenza nel Consiglio direttivo inizia con Paola Barocchi (I995-2002) e Teresa Poggi Salani (I995- 
20I2, vicepresidente 2008-2012), prosegue con Rosanna Bettarini (2008-2012), Ornella Castellani Pollidori (2008-2012), Paola Manni (vicepresidente 2012-2014), Giovanna Frosini accademica segretaria (2017-2020) e Annalisa Nesi accademica segretaria (2020-2023). Severina Parodi è stata la prima donna a ricoprire l'incarico di segretaria. Fino a quel momento e a partire da Bastiano de' Rossi, Accademico segretario che firmò la dedicatoria del Vocabolario del I6r2, questa carica esecutiva di alto profilo era stata sempre ricoperta da uomini. A Nencioni si deve, nel 1997, il primo Consiglio direttivo tutto al femminile.

Le accademiche sono prima di tutto delle studiose che hanno raggiunto considerazione nell'ampio spettro della ricerca sulla lingua; lavorano spesso appartate, contribuiscono in vario modo alla vita dell'Accademia partecipando alle attività dei Centri di ricerca e della Consulenza della lingua italiana contemporanea, alla pubblicazione delle riviste, spesso con ruoli direttivi: attualmente il Centro di grammatica italiana è diretto da Teresa Poggi Salani e l'Archivio procede grazie all'attività di Elisabetta Benucci, studiosa fra l'altro di figure femminili, coadiuvata da altri collaboratori.

Un ricordo particolare va a Severina Parodi che resta nella nostra memoria e nella storia dell'Accademia come simbolo di generosa dedizione allo studio e all'istituzione, di competenza applicata con costanza e sollecitudine nei diversi compiti ai quali era chiamata. Chi entra nel Catalogo degli Accademici, disponibile sul sito della Crusca, trova Severina Parodi, accademica corrispondente dal 1983 - come si è scritto prima - definita semplicemente "studiosa"; a lei si deve lo straordinario contributo alla conoscenza e alla valorizzazione del patrimonio storico dell'istituzione di cui ha fatto parte dal 1964 con mansioni di segreteria e di riordino delle carte antiche, poi come Accademica segretaria dal 1997 al 2003, anno della sua morte. Consulente linguistica e redattrice della "Crusca per voi" fin dalla fondazione della rivista nel ig9o.

Fino dall'inizio abbiamo sottolineato che la Crusca può contare, e ha potuto contare nel tempo, su tante altre donne e, senza voler per forza lasciare da parte gli uomini, più o meno dietro le quinte ne troviamo in ogni settore dell'organigramma. Non potendo nominarle tutte ricordo solo Ada Braschi, insegnante, laureata con Giovanni Nencioni quando era docente al Magistero di Firenze, antesignana e storica collaboratrice dell'Accademia. È stata redattrice e consulente della "Crusca per voi" insieme a Severina Parodi fino dalla fondazione della rivista e ha poi continuato l'attività con Raffaella Setti. Dal passato a oggi, attraverso tante facce di donne che rivediamo ad una scrivania studiare, schedare, preparare articoli, organizzare incontri e convegni, fino al Comitato di redazione tutto al femminile (nove redattrici e una responsabile delle illustrazioni) dell'ultima nata: "Italiano digitale. La rivista della Crusca in rete".

L'Accademia è impegnata, in collaborazione con altre istituzioni, nella valorizzazione del ruolo della donna e certamente non si limita alla ricostruzione della sua storia al femminile attraverso gli approfondimenti biografici delle accademiche. Accademiche e accademici, collaboratrici e collaboratori portano avanti riflessioni e proposte concrete inerenti le problematiche e gli usi della lingua in rapporto al genere, avvalendosi di studiose come Cecilia Robustelli, linguista particolarmente esperta e attiva in questo ambito che collabora da tempo con l'Accademia. Numerose le pubblicazioni, gli incontri e la partecipazione attiva dell'Accademia a iniziative come, ad esempio, la "Giornata internazionale della donna" (20I8), l'"Eredità delle donne" (20I8 e 2020). 
*La citazione è tratta da: Nicoletta Maraschio, Premessa, in Italia linguistica: gli ultimi 150 anni. Nuovi soggetti, nuove voci, un nuovo immaginario, a cura di Elisabetta Benucci e Raffaella Setti, Firenze, Le Lettere, 20II, pp. VII-VIII.

Nello stesso volume il capitolo Le donne e la costruzione della lingua nazionale con i saggi di Cecilia Robustelli, Donne che scrivono tra Otto e Novecento: dalle carte private ai saggi scientifici e di Elisabetta Benucci, «Il piu bel fior ne coglie». Donne accademiche e socie della Crusca.

*Alcune segnalazioni per informazioni e approfondimenti sulle Accademiche, sulle iniziative della Crusca per le donne, sulle collaborazioni:

- Elisabetta Benucci, Letterati alla Crusca nell'Ottocento, Firenze, Accademia della Crusca, $20 r 6$.

- Elisabetta Benucci, Franca Brambilla Ageno e l'Accademia della Crusca: storia di una intensa e proficua collaborazione, "Women Language Literature in Italy / Donne Lingua Letteratura in Italia" II, 2020, pp. 63-82.

- Catalogo degli accademici della Crusca.

- Giovanna Frosini, Donne di Crusca, "Italiano Digitale" 2019, II, 2019/4 (ottobre-dicembre), p. Ioo.

- Guida alla Redazione degli atti amministrativi. Regole e suggerimenti, a cura del gruppo di lavoro promosso da Istituto di teoria e tecniche dell'informazione giuridica e Accademia della Crusca, Ittig - Cnr, 20II.

- Bice Mortara Garavelli, Severina Parodi storica dell'Accademia della Crusca, 28 febbraio 2008, sito web dell'Accademia della Crusca - Marzo 2008.

- Cecila Robustelli, Linee guida per l'uso del genere nel linguaggio amministrativo. Progetto genere e linguaggio. Parole e immagini della comunicazione, in collaborazione con l'Accademia della Crusca, con il finanziamento della Regione Toscana L.R. i6/og Cittadinanza di Genere, 20 s2.

- Cecilia Robustelli, Donne, grammatica e media. Suggerimenti per l'uso dellitaliano, Prefazione di Nicoletta Maraschio Presidente onoraria dell'Accademia della Crusca, Ariccia (RM), Eurograf Sud, 2014.

- Cecilia Robustelli, Sindaco e sindaca. Il linguaggio di genere, Roma, Gruppo editoriale L'Espresso e Accademia della Crusca, 2or6, Postfazione del Presidente dell'Accademia della Crusca Claudio Marazzini.

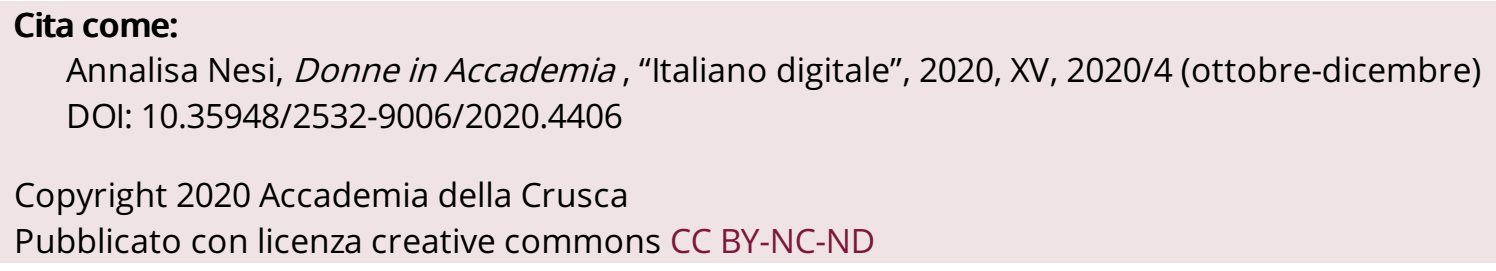

